\title{
Temperature Dependent Surface Resistivity Measures of Commercial, Multiwall Carbon Nanotubes (MWCNT), and Silver Nano-Particle Doped Polyvinyl Alcohol (PVA) Films
}

\author{
Matthew Edwards ${ }^{1 *}$, Stephen Egarievwe ${ }^{2}$, Afef Janen', Tatiana Kukhtarev ${ }^{1}$, \\ Jemilia Polius ${ }^{1}$, John Corda ${ }^{1}$ \\ ${ }^{1}$ Department of Physics, Chemistry \& Mathematics, Alabama A \& M University, Normal, Al, USA \\ ${ }^{2}$ Department of Engineering, Construction Management \& Industrial Technology, Alabama A \& M University, \\ Normal, Al, USA \\ Email: ${ }^{\text {matthew.edwards@aamu.edu }}$
}

Received 27 September 2014; revised 26 October 2014; accepted 6 November 2014

Copyright (C) 2014 by authors and Scientific Research Publishing Inc.

This work is licensed under the Creative Commons Attribution International License (CC BY). http://creativecommons.org/licenses/by/4.0/

CC) (i) Open Access

\begin{abstract}
Pure and doped Polyvinylidene difluoride (PVDF) films, for the detection of infrared radiation, have been well documented using the mechanism of pyroelectricity. Alternatively, the electrical properties of films made from Polyvinyl Alcohol (PVA) have received considerable attention in recent years. The investigation of surface resistivities of both such films, to this point, has received far less consideration in comparison to pyroelectric effects. In this research, we report temperature dependent surface resistivity measurements of commercial, and of multiwall carbon nanotubes (MWCNT), or Ag-nanoparticle doped PVA films. Without any variation in the temperature range from $22^{\circ} \mathrm{C}$ to $40^{\circ} \mathrm{C}$ with controlled humidity, we found that the surface resistivity decreases initially, reaches a minimum, but rises steadily as the temperature continues to increase. This research was conducted with the combined instrumentation of the Keithley Model 6517 Electrometer and Keithley Model 8009 resistivity test fixture using both commercial and in-house produced organic thin films. With the objective to quantify the suitability of PVDF and PVA films as IR detector materials, when using the surface resistivity phenomenon, instead of or in addition to the pyroelectricity, surface resistivity measurements are reported when considering bolometry. We found that the surface resistivity measurements on PVA films were readily implemented.
\end{abstract}

\footnotetext{
${ }^{*}$ Corresponding author.
}

How to cite this paper: Edwards, M., et al. (2014) Temperature Dependent Surface Resistivity Measures of Commercial, Multiwall Carbon Nanotubes (MWCNT), and Silver Nano-Particle Doped Polyvinyl Alcohol (PVA) Films. Materials Sciences and Applications, 5, 915-922. http://dx.doi.org/10.4236/msa.2014.513093 


\section{Keywords}

\section{Silver Nano-Particles, Surface Resistivity, Multi-Wall Carbon Nanotubes (MWCNT), Polyvinyl Alcohol (PVA) Films}

\section{Introduction}

Applications of dielectric films based on properties of such materials have been an important topic in electronics and the semiconductor industry for scores of years. Moreover, predicting the level of electrical currents in such materials has been desirable in devices ranging from metal-oxide-semiconductor field effect transistor (MOSFET) in integrated circuits (ICs) to infrared radiation (IR) detectors [1]-[3]. In addition to bulk property considerations, understanding the surface properties [4] [5] of such films has become extremely important in recent years, occurring simultaneously as MOSFETs have become much smaller in size. To that extent, the development of appropriate surface configured thin films and testing the electrical current on the surfaces of such films have become the focus of this investigation.

We have determined the surface resistivity characterization of PVA dielectrics to be a particularly important physical phenomenon to investigate owing to its probable use in sensor science or even in IR detection likening to that of the PVDF material that we have studied previously [6]-[9]. Moreover, in this study, we have included measurements on commercial and doped PVA films, with either MWCNT or Ag-nanoparticles as dopants [10] [11], while no measurements have been reported here on PVDF films.

\section{Theory of Electrical Conduction in Dielectrics}

A dielectric is a material in which electrons are very tightly bonded to nuclei, but not so tightly bonded that some energetic electrons cannot be detached from nuclei at higher temperatures [12]-[18]. Moreover, through the dielectric polarization, the electric charges in dielectrics do respond to applied electric field and can produce electric currents in such fields. In this regard, dielectric materials are nearly insulators in which the electrical bulk and surface resistivity are extremely large and the energy band gaps, as well, are also corresponding large. In general, the value of energy band gap of insulators is established to be larger than $3 \mathrm{eV}$ to $5 \mathrm{eV}$ [3] [14]. Although not all dielectrics are insulators, all insulators are typical dielectrics, and at $0 \mathrm{~K}$, the conduction band is empty and the valence band is filled. In this regard, there is no carrier for electrical conduction. When the temperature is larger than $0 \mathrm{~K}$, some electrons will be thermally excited from the valence band and also from any existing donor impurity level to the conduction band. These electrons, the thermally excited electrons, will contribute to the current transport of the dielectric material. Similarly, holes will be generated by acceptor impurities and excited electrons in the valence band should leave vacancies. The conduction current of insulators at normal applied electric field will be very small because their bulk resistivities are inherently large, on the order of $10^{8}$ to $10^{20} \Omega \cdot \mathrm{cm}$; however, dielectric conduction current is observable when a relatively large electric field is applied. These observable conduction currents owe their existence to one of more of a large number of different conduction mechanisms, and the conduction currents can be critical for the application of the dielectrics in electrical devices. For example, the tunneling dielectric of flash memories, the gate dielectric of MOSFETs, and the capacitor dielectric of dynamic random access memories are all of primary importance to IC applications. In each of these cases, the conduction current must be lower than a certain level to meet the specific reliability criteria under normal operation of the devices.

To measure the observed current through dielectric films or on the surface of the films, one must use a sample testing device. Presently, two types of device structures are used in sample testing: one type is metal-insulatormetal structure, which is called the MIM capacitor or the MIM diode, and the second kind is the metal-insulator-semiconductor (MIS) capacitor [3]. In the MIM capacitor, the asymmetry of the electrical properties, when the top and bottom electrodes are made of different metals, is a feature that must be considered, since different metals generally lead to different work functions, and thus result in different metal-dielectric interface barriers. The main parameters in this type of measurement are the barrier height of the metal-dielectric interface and the effective mass of the conduction carriers. The MIS capacitor, the second type for sample testing, is the most useful device in the study of dielectric surfaces. Moreover, the structure of the MIS capacitor is inherently 
asymmetric, and the user must be cautious about the voltage drop across the layers. If the MIS capacitor can be biased in such a way that the semiconductor surface is in accumulation of charges, the voltage drop across the semiconductor is minimal, and most of the voltage will be applied across the dielectric film; but, if the semiconductor surface is in a depletion or inversion condition, a voltage drop will occur across the semiconductor and then this voltage drop needs to be considered in calculations of the electric field across the dielectric film. The testing device of this investigation is the MIM capacitor type, consisting of the combined Keithley electrometer and Keithley resistivity test fixture.

Among the currently existing conduction mechanisms, some have been determined to depend on the electrical properties at the electrode-dielectric contact and others depend on the bulk-limited conduction mechanisms or transport-limited conduction mechanisms of the material itself. Table 1 gives some important conduction mechanisms in dielectrics. The methods to distinguish these conduction mechanisms are essential because there are several conduction mechanisms that may all contribute to the conduction current through the dielectric or on its surface at the same time; however, measuring the temperature dependent conduction currents may afford us a useful method to know the constitution of the conduction currents, since several conduction mechanisms depend on the temperature in different ways.

The electrode-limited conduction mechanisms are thought to develop from the electrical properties at the electrode-dielectric contact, and the most important parameter in this type of conduction is the barrier height that occurs at the electrode-dielectric interface [6] [7]. The current due to thermionic emission is highly dependent on the temperature, but the tunneling current is nearly temperature independent. In addition to the barrier height at the electrode-dielectric interface, the effective mass of the conduction carriers in dielectric films is also an important factor in the electrode-limited conduction mechanisms. On the other hand, the bulk-limited conduction mechanisms are thought to develop from the electrical properties of the dielectric itself. The most significant parameter in this type of conduction mechanism is the trap energy levels in the dielectric films. Owing to the bulk-limited conduction mechanisms, some important electrical properties in the dielectric films can be extracted, such as the trap spacing, the trap energy level, the trap density, the electronic drift mobility, the dielectric relaxation time, and the density of states in the conduction band.

\section{Experimental and Methods}

\subsection{Sample Preparation}

\subsubsection{Making PVA Thin Films}

The method to prepare a $4 \%$ polyvinyl alcohol solution as a processor to the PVA thin film, the following steps are used: Heat $100 \mathrm{ml}$ of water or water and ethyl alcohol $(50 / 50)$ to $88^{\circ} \mathrm{C}-90^{\circ} \mathrm{C}$, in a beaker to just under the boiling point of water. Once the solvent is heated, lightly sprinkle 4 grams of the polyvinyl alcohol into the solvent while continuing magnetic stirring. Add the polyvinyl alcohol particles slowly so that each particle is "wetted" thus allowing each particle to go into solution, while still stirring magnetically and being cautious to not overheat the mixture (Overheating the mixture will cause the solvent to evaporate too quickly, and an undesirable thick gooey mass of wet polymer will settle out and stick to the wall of the beaker). It takes about 15 minutes to make the $100 \mathrm{ml}$ gel-like solution. Next, the dopant is added as needed and the mixture is dispersed into a

\begin{tabular}{ll}
\hline \multicolumn{1}{|c}{ Table 1. Important conduction mechanisms in dielectrics. } \\
\hline \multicolumn{1}{|c}{ Electrode-Limited Conduction Mechanisms } & \multicolumn{1}{c}{ Bulk-Limited Conduction Mechanisms } \\
\hline 1. Schottky emission & 1. Poole-Frenkele emission \\
2. Fowler-Nordheim tunneling & 2. Hopping conduction \\
3. Direct tunneling & 3. Ohmic conduction \\
4. Thermionic-field emission & 4. Space-charge-limited conduction \\
& 5. Ionic conduction \\
& 6. Grain-boundary-limited conduction
\end{tabular}

Source: Reference [3]. 
pre-selected size petri disks to allow the films to form, which takes more than 3 days. As determined, the dopant in the solution can be detected as is done for Ag nano-particle in the uv-visible spectrograph in Figure 1(b). In this regard, dielectric thin films samples have been prepared with the solvent evaporation procedure as shown in Figure 1(a) and Figure 1(b).

\subsubsection{Making Large PVDF Thin Films}

The PVDF thin film are prepared from casting a gel-like solution of PVDF in a mixture with methyl ethyl ketone (MEK) and allowed to dry followed by pressing the precursor material to release stress when achieving large diameter PVDF thin films [7]-[20].

\subsection{Experimental Setup}

In addition, for testing purposes, we selected as the measurement system the combined Keithley Model 6517 Electrometer and Keithley Model 8009 resistivity test fixture with the Amprobe wireless thermometer when using the J-type thermocouple as shown in Figure 2(a) and Figure 2(b). Humidity, another important factor in surface resistivity measurements, was monitored with a commercial grade humidifier. In this research, we specified humidity as normally low, normally medium or normally high. All of our reported measurements were made at normal medium humidity at approximately $70 \%$.

What is the relevant feature to measure the surface resistivity using the combined Keithley electrometer and Keithley test fixture? Here, we give a brief review of the procedure: for all such conducting dielectric materials, the relations between surface resistivity and the surface current for Keithley concentric ring probe test fixture, a surface current density is defined in the area between the two rings [4] [5] [19]. Then through knowing the surface current density, it is possible to find the electric field between the electrodes rings. In this regard, the surface current density $J_{s}$ for this circular configuration is determined as

$$
J_{s}=\frac{I_{s}}{C}=\frac{I_{s}}{2 \pi r}
$$

where $C$ is the total perpendicular length to the radial current $I_{s}, r$ is the radius of total the perpendicular circle, varying from the inner electrode radius $R_{1}$ to outer electrode radius $R_{2}$. It is assumed that all the currents flow between the electrodes along the surface and do not penetrate into the bulk of the material. Ohm's law describes the relationship between the surface current density $J$, the surface resistivity $\rho_{s}$ and the surface electric field $E$ as

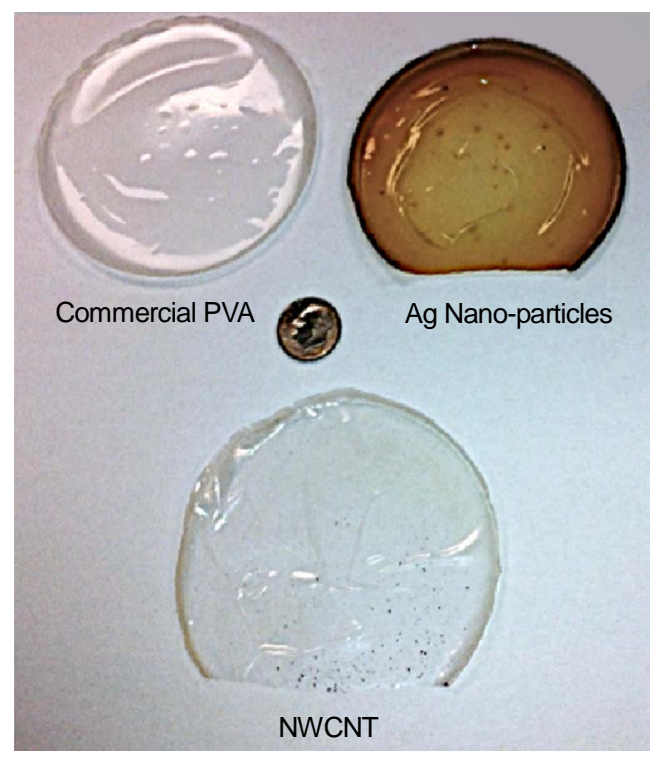

(a)

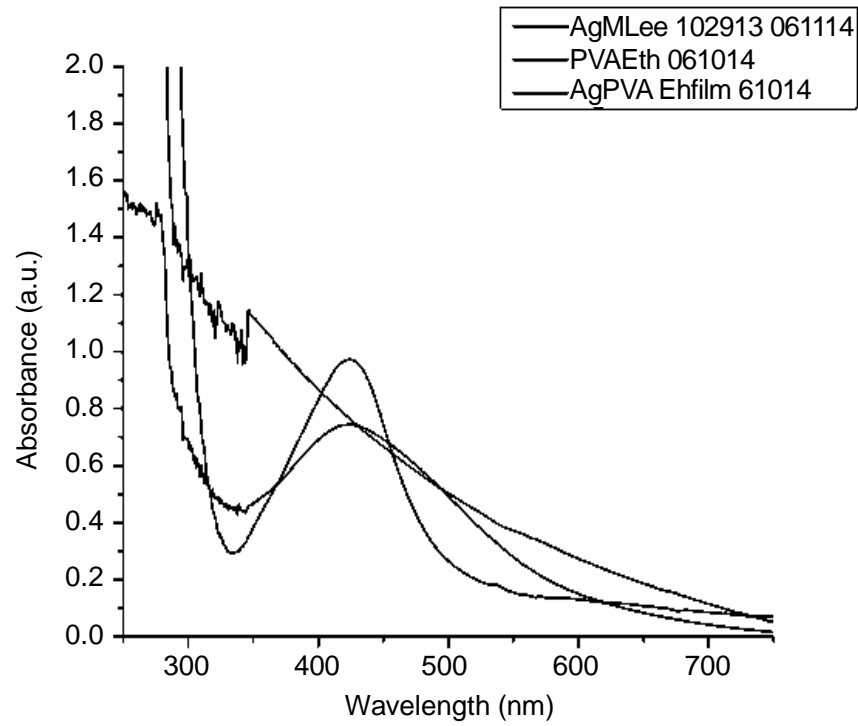

(b)

Figure 1. (a) Image of samples; (b) UV-VIS spectrograph of Ag-nano-particles in PVA film. 


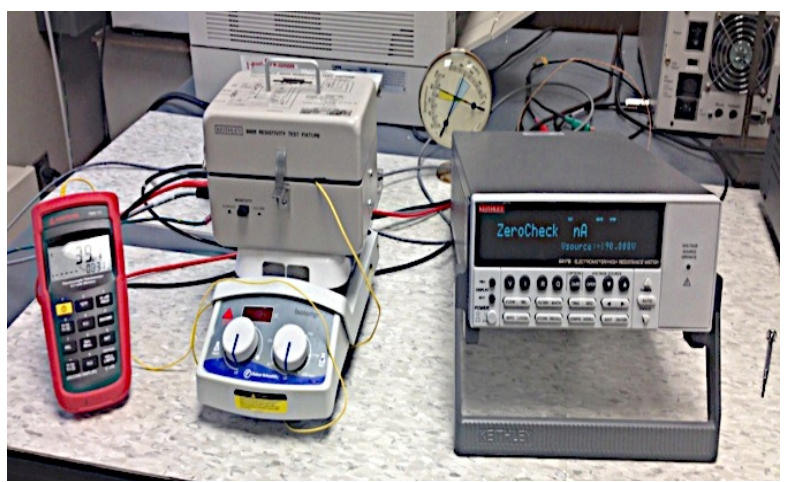

(a)

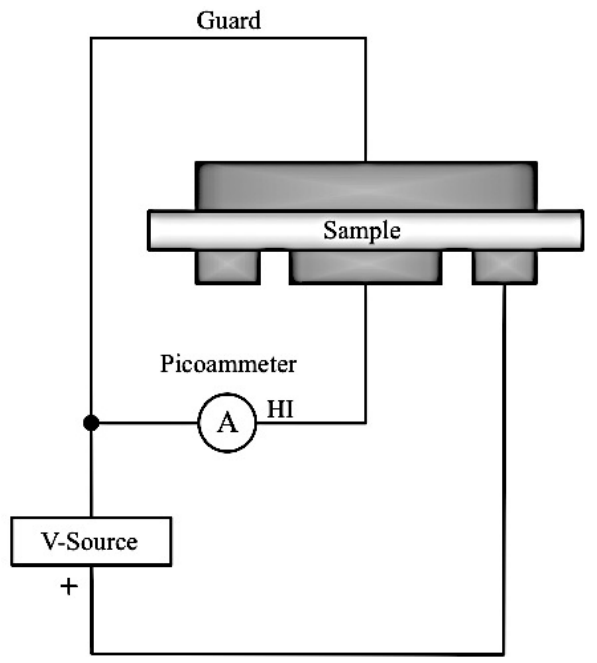

(c)

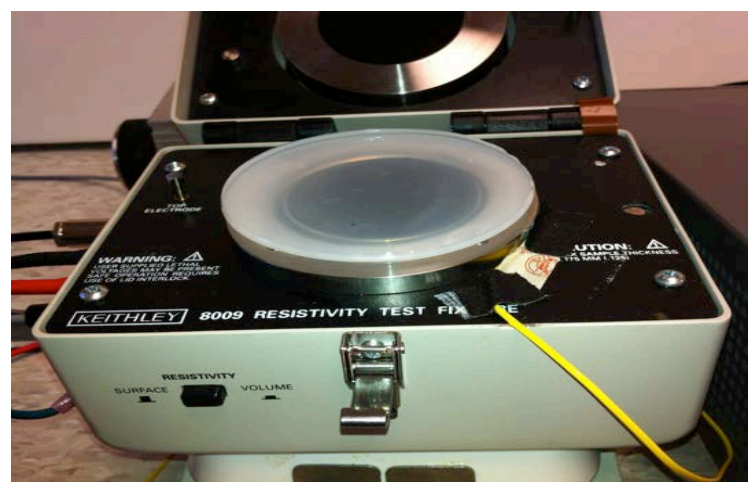

(b)

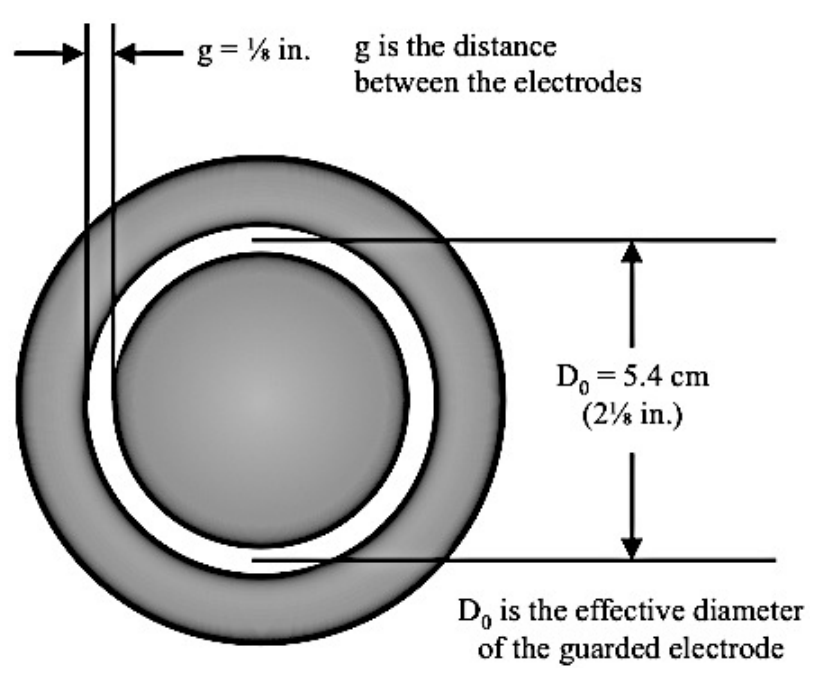

(d)

Figure 2. (a) Experimental setup; (b) Interior view of fixture with commercial PVA film; (c) Illustration of current path with sample and electrodes; (d) Close-up showing circular electrodes.

$$
J_{s}=\frac{\frac{V}{L}}{\rho_{s}}=\frac{E}{\rho_{s}}
$$

where $L$ is the radial distance between the two concentric electrodes. Now, when combining Equation (1) and (2), the voltage $V$ between the electrodes is given by

$$
V=\int_{R_{1}}^{R_{2}} E \mathrm{~d} r=\int_{R_{1}}^{R_{2}} \frac{\rho_{s} I_{s}}{2 \pi r}=\frac{\rho_{s} I_{s}}{2 \pi} \ln \left(\frac{R_{2}}{R_{1}}\right)
$$

Finally, upon solving for the surface current $I_{s}$ with $R_{1}=57.2 \mathrm{~mm}$ and $R_{2}=85.7 \mathrm{~mm}$, we get that

$$
\rho_{s}=\frac{2 \pi}{\ln \left(\frac{R_{2}}{R_{1}}\right)} \frac{V}{I_{s}}=53.4 \frac{V}{I_{s}} \mathrm{ohm}
$$

Thus, the measurement of $I_{s}$ for a given voltage $V$ gives a method to obtain the surface resistivity, $\rho_{s}$.

Through using the combine Keithley electrometer and Keithley resistivity test fixture, we have measured surface resistivities as a function of temperature in a limited range above room temperature. Resistivity measurements were made on commercial PVA thin films, on the Ag-nanoparticle or the MWCNT doped polyvinyl alcohol (PVA) thin films, and on the copier-paper thin films. Our measurements indicated significant temperature 
dependence for each sample type. The results showed a large variation in the resistivity with a characteristic behavior of decreasing in resistivity in the lower temperatures to a minimal value followed by a rapid increase in resistivity from that point forward to the end of our measurement range. Also, we have determined cubic temperature dependence from fitting the data. The observed variations are more pronounced with Ag-nanoparticles and MWCNT doped thin films as compared to commercial PVA and copier-paper thin films. All resistivity values of our PVDF films, in our present experimental setup, were too high relative to doped PVA for accurate measurements. Figures 2(a)-2(c) show our setup, consisting of a combined Keithley electrometer and Keithley test fixture, with the Amprobe wireless thermometer. Figures 3(a)-3(d) and Table 2 give the observed temperature dependent measurements.

\section{Results and Discussion}

Figures 3(a)-3(d) show the temperature dependent surface resistivity measurements of PVA for the commercial films, the doped Ag nano-particles or the doped MWCNT and the copier paper films. All of the resulting graphs have the same characteristic behavior: the surface resistivity is only slightly high at low temperatures, but decreases initially as the temperature increases reaching a minimal value where upon the surface resistivity increases as the temperature continues to increase to our experimental limit of $40^{\circ} \mathrm{C}$. No temperature dependent measurements were possible on PVDF films in our existing measurement configuration, thus realizing an upper limit $10^{14} \Omega$ or higher. Temperature sensors are readily suggested by our preliminary data with PVA thin films, but additional considerations are needed to consider other sensor-types. Also, the production of larger PVDF thin films (those greater that 2.5" in diameter) that are free from internal stress has been elusive to our effort but remains a task to be completed. Also, we have the added task of being able to achieve uniform dispersion of dopants in the mixture during the film formation phase.

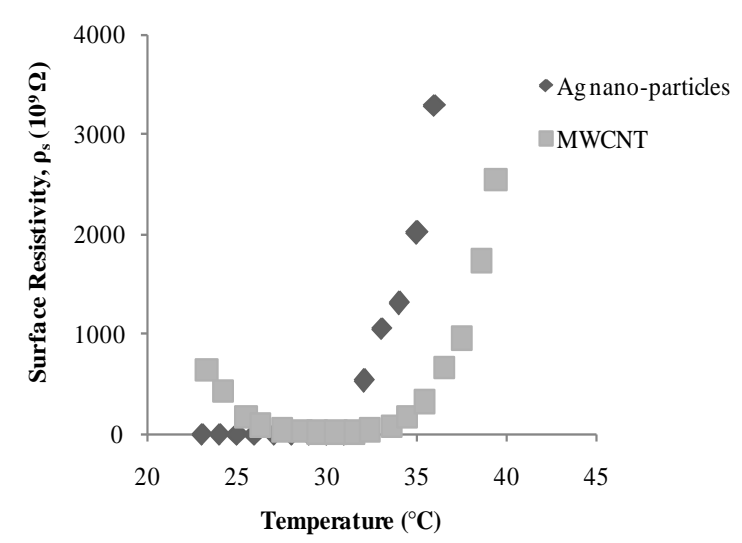

(a)

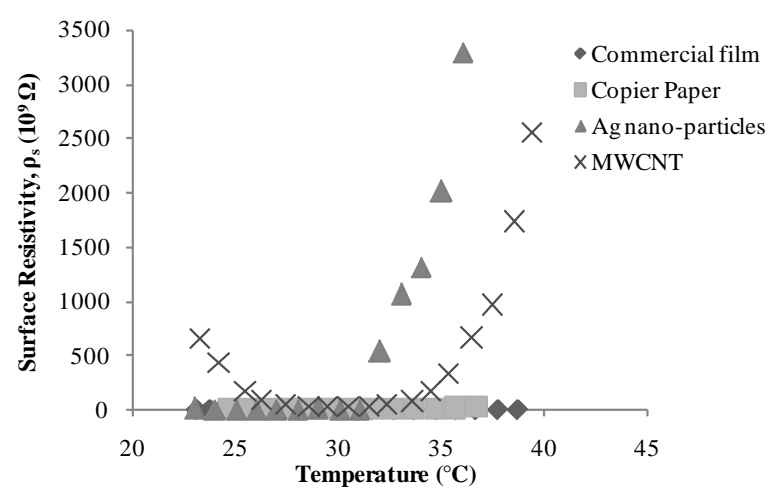

(c)

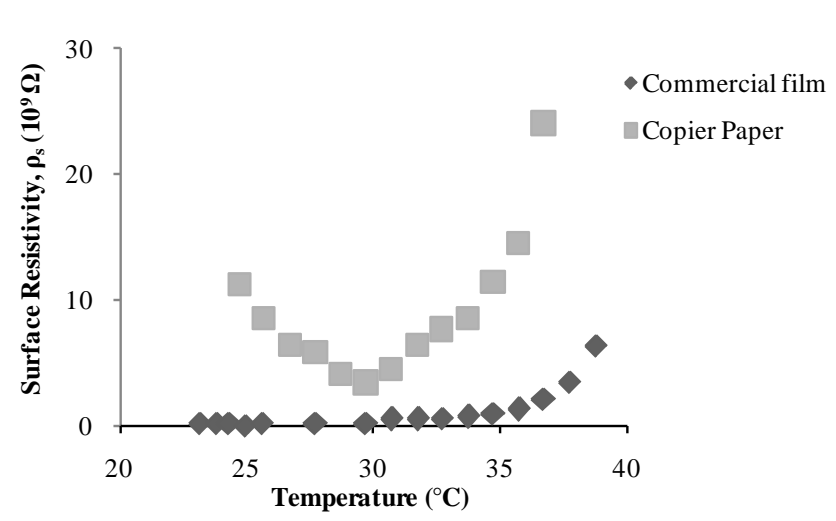

(b)

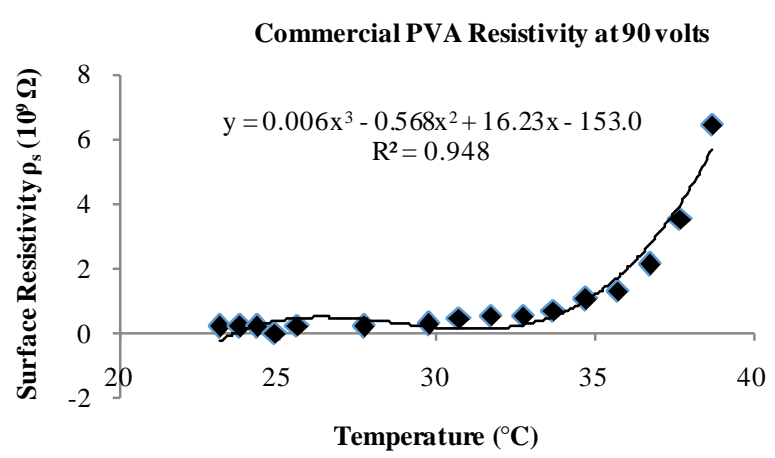

(d)

Figure 3. (a) Surface resistivity graphs of commercial PVA film and copier-paper; (b) Surface resistivity graphs of Ag nanoparticle and MWCNT; (c) Surface resistivity of combined samples; (d) Surface resistivity commercial PVA film with curve fitting. 
Table 2. Surface resistivity data for commercial PVA films, Ag nano-particles PVA films and MWCNT PVA films.

\begin{tabular}{cccccccc}
\hline $\begin{array}{c}\text { Temp } \\
\left({ }^{\circ} \mathrm{C}\right)\end{array}$ & $\begin{array}{c}\text { Surface Resistivity } \\
\text { Commercial PVA }\left(10^{9} \Omega\right)\end{array}$ & $\begin{array}{c}\text { Temp } \\
\left({ }^{\circ} \mathrm{C}\right)\end{array}$ & $\begin{array}{c}\text { Surface Resistivity } \\
\text { Ag-Nano-particle }\left(10^{9} \Omega\right)\end{array}$ & $\begin{array}{c}\text { Temp } \\
\left({ }^{\circ} \mathrm{C}\right)\end{array}$ & $\begin{array}{c}\text { Surface Resistivity } \\
\text { MWCNT }\left(10^{9} \Omega\right)\end{array}$ & $\begin{array}{c}\text { Temp } \\
\left({ }^{\circ} \mathrm{C}\right)\end{array}$ & $\begin{array}{c}\text { Surface Resistivity } \\
\text { Paper } 10^{9} \Omega\end{array}$ \\
\hline 23.1 & 0.21 & 23 & 15.02 & 23.3 & 645.97 & 24.7 & 11.18 \\
23.8 & 0.21 & 24 & 5.52 & 24.2 & 431.03 & 25.7 & 8.58 \\
24.3 & 0.21 & 25 & 3.41 & 25.5 & 173.50 & 26.7 & 6.41 \\
24.9 & 0.00 & 26 & 2.63 & 26.3 & 93.14 & 27.7 & 5.79 \\
25.6 & 0.24 & 27 & 3.43 & 27.5 & 42.42 & 28.7 & 4.07 \\
27.7 & 0.21 & 28 & 5.11 & 28.6 & 28.52 & 29.7 & 3.41 \\
29.7 & 0.29 & 29 & 13.35 & 29.5 & 23.39 & 30.7 & 4.53 \\
30.7 & 0.50 & 30 & 5.17 & 30.5 & 24.58 & 31.7 & 6.41 \\
31.7 & 0.55 & 31 & 10.45 & 31.5 & 23.33 & 32.7 & 7.63 \\
32.7 & 0.57 & 32 & 538.79 & 32.4 & 41.43 & 33.7 & 8.58 \\
33.7 & 0.73 & 33 & 1065.63 & 33.6 & 78.27 & 34.7 & 11.44 \\
34.7 & 1.05 & 34 & 1313.11 & 34.5 & 168.63 & 35.7 & 14.56 \\
35.7 & 1.30 & 35 & 2010.88 & 35.4 & 322.55 & 36.7 & 24.03 \\
36.7 & 2.14 & 36 & 3291.78 & 36.5 & 658.36 & & \\
37.7 & 3.56 & & 37.5 & 961.20 & & \\
38.7 & 6.43 & & 38.6 & 1728.78 & & \\
& & & & 39.4 & 2542.86 & &
\end{tabular}

\section{Conclusions}

The preliminary experimental results obtained from this investigation can be summarized as:

- PVA thin films, doped and commercial, can be developed with the solvent evaporation method.

- PVDF thin films can be developed from the solution casting technique, yet the production of larger, stress free PVDF films, remains as an ongoing task for us.

- The combined system of Keithley Model 6517 electrometer, Keitley Model 8009 test fixture and the amprobe thermometer can be used readily to measure surface resistivity of commercial PVA films, Ag nano-particle or MWCNT doped PVA films and copier-paper films, but cannot in the present configuration be used to measure the surface resistivity of PVDF films.

- Surface resistivity of Ag nano-particle doped PVA films is generally higher in value than that of commercial PVA and varies the most in the temperature range from $22^{\circ} \mathrm{C}$ to $40^{\circ} \mathrm{C}$, followed in order of decreasing to MWCNT and copier-paper.

- A cubic polynomial fits our data extremely well, thus suggesting the existence of competing conduction mechanisms during temperature changes in such dielectrics.

- The application surface resistivity of PVA as a temperature sensor is readily apparent, but its application as other sensor-types remains to be determined.

- Further study is needed on PVA to ascertain the surface resistivity temperature dependence in other temperature ranges, and to determine conduction mechanisms that are involved as a function of temperature.

- Further study is needed on PVDF films to ascertain surface resistivity properties more conclusively that what are reported here.

\section{Acknowledgments}

Thanks goes to DHS grant 2012-DN-077-ARI065-04, HBCU-UP grant HRD-0928904 and the Evans Allen grant for partial funding of this research, to Ms. Sheral Robinson for graphic support, and to Dr. Mohan Aggar- 
wal for continued support of our research agenda..

\section{References}

[1] Malikov, E.Y., Muradov, M.B., Akperov, O.H., Eyvazova, G.M., Puskas, R., Madarasz, L., Kukovecz, A. and Konya, Z. (2014) Synthesis and Characterization of Polyvinyl Alcohol Based Multiwalled Carbon Nanotube Nanocomposites. Physica E: Low-dimensional Systems and Nanostructures, 61, 129-134. http://dx.doi.org/10.1016/j.physe.2014.03.026

[2] Renoux, P., Jonsson, S.A., Levente, K.J., Hamann, H.F. and Ingvarsson, S. (2011) Sub-Wavelength Bolometers: Uncooled Platinum Wires as Infrared sensors. Optics Express, 19, 8721. http://dx.doi.org/10.1364/OE.19.008721

[3] Chiu, F.-C. (2014) A Review on Conduction Mechanisms in Dielectric Films. Advances in Materials Science and Engineering, 2014, Article ID: 578168.

[4] Maryniak, W.A., Uehara, T. and Noras, M.A. (2003) Surface Resistivity and Surface Resistance Measurements Using a Concentric Ring Probe Technique. Trek Application Note No. 1005.

[5] Wong, C.K. and Shin, F.G. (2005) Electrical Conductivity Enhanced Dielectric and Piezoelectric Properties of Ferroelectric 0-3 Composites. Journal of Applied Physics, 97, 064111. http://dx.doi.org/10.1063/1.1862317

[6] Nye, J.F. (1985) Physical Properties of Crystals. Oxford Science Publications.

[7] Edwards, M.E., Batra, A.K., Ashwith, C.K., Guggilla, P. and Curley, M. (2012) Pyroelectric Properties of PVDF: MWCNT Nanocomposite Film for Uncooled Infrared Detectors. Materials Sciences and Applications, 3, 851-855. http://dx.doi.org/10.4236/msa.2012.312124

[8] Navid, A., Lynch, C.S. and Pilon, L. (2010) Purified and Porous Poly (Vinylidene Fluoride-Trifluoroethylene) Thin Films for Pyroelectric Infrared Sensing and Energy Harvesting. Smart Materials and Structures, 19, 1-13. http://dx.doi.org/10.1088/0964-1726/19/5/055006

[9] Hartono, A., Satira, S., Djamal, M. and Ramli, E.S. (2013) Poly (Vinylidene Fluoride) Thin Film Prepared by Roll Hot Press. Journal of Applied Physics, 3, 7-11.

[10] Prathna, T.C., Chandrasekaran, N., Raichur, A.M., Mukherjee, A. (2011) Biomimetic Synthesis of Silver Nanoparticles by Citrus limon (Lemon) Aqueous Extract and Theoretical Prediction of Particle Size. Colloids and Surfaces B: Biointerfaces, 82, 152-159. http://dx.doi.org/10.1016/j.colsurfb.2010.08.036

[11] Malikov, E.Y., Muradov, M.B., Akperov, O.H., Eyvazova, G.M., Puskas, R., Madarasz, D., Nagy, L., Kukovecz, Á. and Kónya, Z. (2014) Synthesis and Characterization of Polyvinyl Alcohol Based Multiwalled Carbon Nanotube Nanocomposites. Physica E, 61, 129-134. http://dx.doi.org/10.1016/j.physe.2014.03.026

[12] Reitz, J.R., Milford, F.J. and Christy, R.W. (1980) Foundation of Electromagnetic Theory. Addison-Wesley Publishing Co., Boston.

[13] Agullo'-Popez, F., Cabrera, J.M. and Agullo’-Rueda, F. (1994) Electrooptics: Phenomena, Materials, and Applications. Academic Press, Waltham.

[14] Kittel, C. (2005) Introduction to Solid State Physics. John Wiley and Sons, Hoboken.

[15] Hinchliffe, A. and Munn, R.M. (1985) Molecular Electromagnetism. John Wiley and Sons, Hoboken.

[16] Griffiths, D.J. (1999) Introduction to Electrodynamics. 3rd Edition, Prentice Hall, Upper Saddle River.

[17] Israelachili, J.N. (1985) Intermolecular and Surface Forces, with Applications to Colloidal and Biological Systems. Academic Press, Waltham.

[18] Borch, J., Lyne, M., Mark, R. and Habeger Jr., C. (2010) Handbook of Physical Testing of Paper. CRC Press, Boca Raton.

[19] Li, Q., Xue, Q.Z., Gao, X.L. and Zheng, Q.B. (2009) Temperature Dependence of the Electrical Properties of the Carbon Nanotube/Polymer Composites. eXPRESS Polymer Letters, 3, 769-777.

[20] Siemann, U. (2005) Solvent Cast Technology—A Versatile Tool for Thin Film Production. Progress in Colloid and Polymer Science, 130, 1-14. 
Scientific Research Publishing (SCIRP) is one of the largest Open Access journal publishers. It is currently publishing more than 200 open access, online, peer-reviewed journals covering a wide range of academic disciplines. SCIRP serves the worldwide academic communities and contributes to the progress and application of science with its publication.

Other selected journals from SCIRP are listed as below. Submit your manuscript to us via either submit@scirp.org or Online Submission Portal.
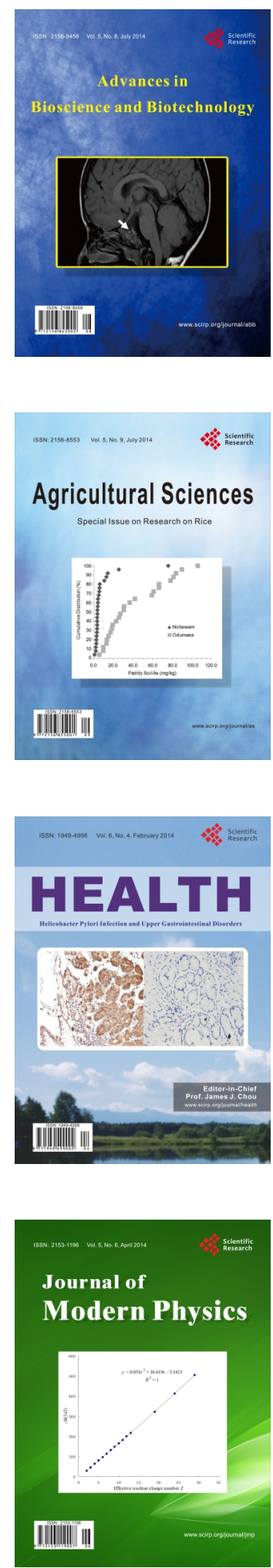
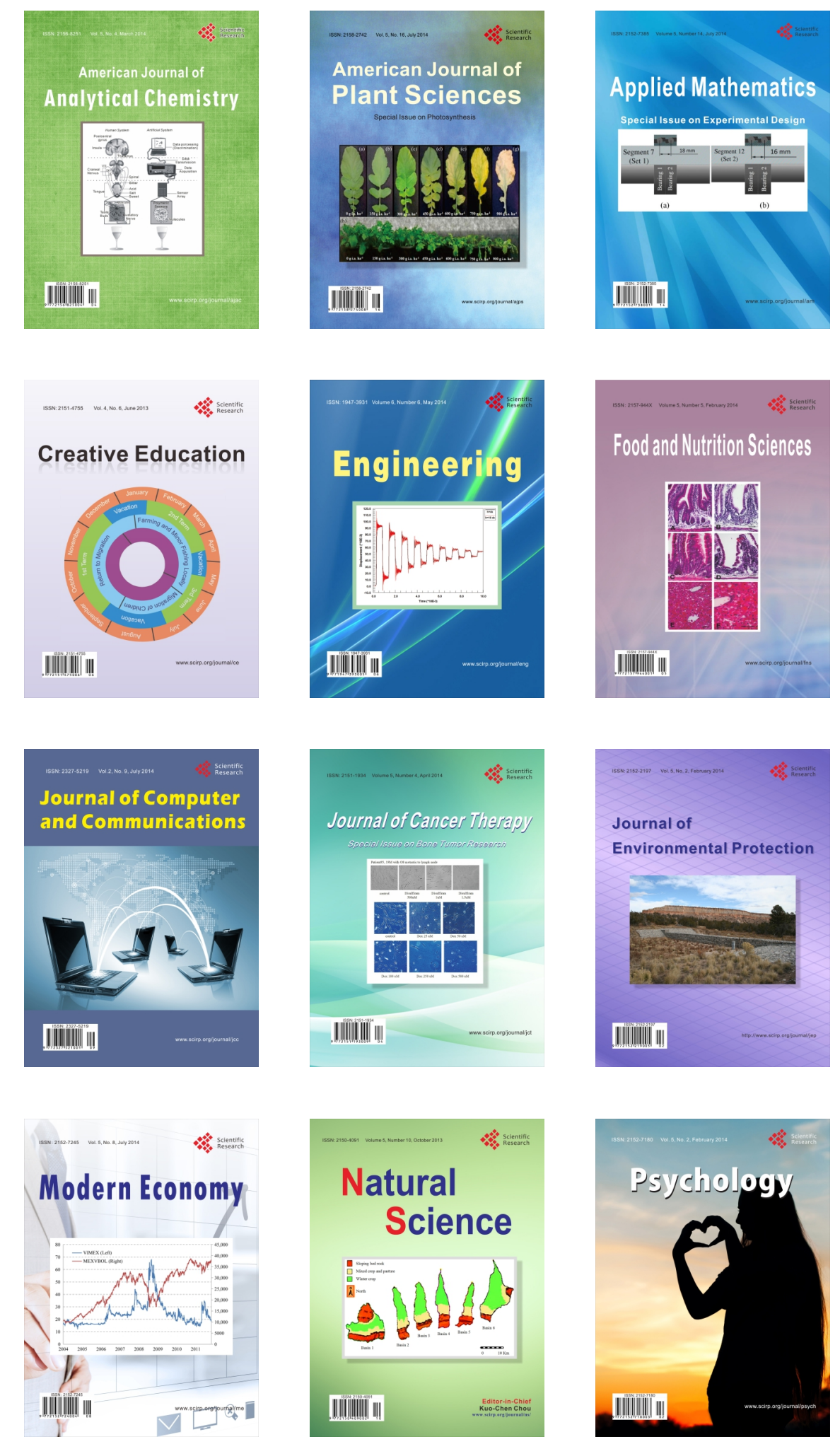